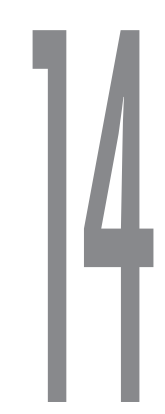

\title{
PLANIFICACIÓN DEL TIEMPO DE TRABAJO CON CUENTAS DE HORAS: EL CASO INDUSTRIAL*
}

\author{
ALBERT COROMINAS SUBIAS \\ AMAIA LUSA GARCÍA \\ JORDI OLIVELLA NADAL \\ INSTITUTO DE ORGANIZACIÓNY CONTROL DE SISTEMAS INDUSTRIALES \\ DEPARTAMENTO DE ORGANIZACIÓN DE EMPRESAS \\ UNIVERSIDAD POLITÉCNICA DE CATALUÑA
}

Palabras clave: Programación, horarios, cuentas de horas.

\section{Introducción}

Los problemas de planificación del tiempo de trabajo han generado un volumen importante de literatura (Ernst et al., 2004). A los problemas clásicos se han añadido los generados por la flexibilidad a largo plazo, que se concretan en los que incluyen la anualización y las cuentas de horas. Estos problemas han sido tratados en diversos trabajos realizados en el desarrollo del mismo proyecto de investigación al que pertenece el presente trabajo (Corominas et al., 2002; Corominas et al., 2005; Corominas et al., 2006; Corominas et al., 2007 a y b; Pastor y Olivella, 2008), así como por otros autores (Hung, 1999; Azmat et al., 2004). Aquí se estudia, concretamente, la planificación con cuentas de horas en el caso industrial.

En la cuenta de horas se anotan las horas trabajadas por encima o por debajo de un valor de referencia; el saldo de la cuenta indica las horas que la empresa debe al trabajador o las que el trabajador debe a la empresa. Las cuentas de horas aparecen primeramente para ofrecer flexibilidad a los trabajadores
(Owen, 1977). Esta flexibilidad está, generalmente, condicionada a que no resulte perjudicial para la actividad. Aquí se considera, sin embargo, el caso de flexibilidad por necesidades de la empresa. Esta modalidad fue desarrollada inicialmente en Alemania, en el sector del automóvil, y desarrollada después en otros sectores y países (Lehndorff, 1999).

El objeto de las cuentas de horas para necesidades de la producción es asignar horas de trabajo por encima o por debajo de las contratadas para cubrir las fluctuaciones de la demanda. Las diferencias se compensan más adelante, sin que exista un límite para esta compensación. Esta modalidad genera un problema de planificación claramente diferenciado. Hay que indicar que algunos casos en que se prevé tiempo extra de trabajo a recuperar posteriormente, o al revés, pueden ser asimilados al caso de la cuenta de horas, aunque en la regulación que se utilice no se haya definido explícitamente la cuenta.

\section{La modalidad de cuentas de horas en el sector industrial}

Una cuenta de horas es una herramienta de gestión de los recursos humanos que considera las diferen-

\footnotetext{
*Financiado por el proyecto DPI2004-05797 del MEyC y FEDER.
} 
cias entre las horas contractuales y las trabajadas de un empleado, que han de ser equilibradas en el futuro. Aquí se trata el caso en que las variaciones son debidas a necesidades de la empresa. Los acuerdos colectivos que establecen cláusulas de flexibilidad por razones empresariales fueron desarrollados inicialmente en Alemania y posteriormente se extendieron por toda Europa (Lehndorff, 1999). En España, se han establecido acuerdos de aplicación de esta modalidad en factorías de las empresas Renault (DGT, 2004), Opel (SPE, 2004), Seat (DGT, 2006) y Sony (S. del A., 2007).

El caso industrial se diferencia muy fuertemente de los casos de servicios. Por un lado, los inventarios se utilizan, en mayor o menor medida, lo que, cuando menos, hace innecesarias las fluctuaciones de capacidad durante el mismo día que se dan en los clásicos problemas de cobertura de una carga de trabajo en los servicios. Bien al contrario, en la industria se dan unos ritmos de fabricación, en general difícilmente modificables, que exigen a su vez un determinado volumen de trabajadores disponibles. En estos casos el volumen de producción viene determinado por el tiempo operativo (modelos muy flexibles como algunas aplicaciones de la manufactura celular quedan fuera del ámbito de aplicación de este trabajo).

\section{Descripción del problema}

Las características del problema se resumen a continuación:

- Todos los trabajadores del colectivo trabajan las mismas horas.

- Se conoce una previsión de la demanda de los diferentes productos.

- Se define una productividad horaria para cada producto.

- Existen inventarios iniciales y tras los distintos periodos, de cada producto. El mantenimiento en almacén de los inventarios genera un coste.

- Se consideran cuentas de horas iniciales individuales para tener en cuenta las diferencias debidas a la antigüedad y a las bajas laborales y otras ausencias.

- Existen periodos de vacaciones en los que se interrumpe la fabricación pero no el suministro desde almacén.
- La demanda no servida a tiempo se pierde.

- Se define un número de horas, por período, que es el valor de referencia, comprendido en un intervalo cuyo valor mínimo se establece con el fin de evitar jornadas excesivamente cortas. La diferencia entre el número de horas trabajadas, dentro de este intervalo, y el valor de referencia, se anota en la cuenta de tiempo de trabajo. El tiempo de trabajo por período (acotado superiormente, por supuesto), puede ser superior al valor máximo del mencionado intervalo, pero la diferencia entre las horas trabajadas y dicho valor máximo no se anota en la cuenta sino que tiene la consideración de horas extra y se retribuye consecuentemente.

- Existe un máximo y un mínimo para el valor del saldo de las cuentas de horas de los trabajadores. Cuando se ha alcanzado el mínimo (el grupo de trabajadores «debe» un gran número de horas a la empresa), las horas por debajo del valor de referencia no se computan en el saldo (se «perdonan») para evitar que se programen innecesariamente jornadas con un número de horas superior al valor de referencia sólo para que el saldo de los trabajadores de dicho grupo se mantenga en los límites (las horas perdonadas se denominan underaccount). Cuando se ha alcanzado el máximo, las horas que se realizan por encima del valor de referencia (denominadas overaccount) se pagan a un precio especial y no computan en el saldo. Hay un límite anual para estas últimas horas.

- Se establece una cota inferior y una cota superior del saldo global al final del horizonte de planificación, en previsión de las necesidades de periodos posteriores.

- En tanto que mejore la solución, se incluye la posibilidad de que horas que podrían ser incluidas en las cuentas de horas pasen a ser consideradas underaccount o overaccount.

El problema ha sido modelizado como un programa lineal mixto. La función objetivo a minimizar se corresponde con el coste más un factor de penalización de las horas perdonadas (underaccount). Se incluye como objetivo secundario que al final del horizonte de planificación los saldos sean preferentemente positivos o negativos, según las necesidades futuras previstas. 


\section{Modelo}

Aunque todos los trabajadores realizan las mismas jornadas y se podrían tratar conjuntamente, el hecho de que no todos ellos partan de la misma situación, en relación con sus saldos iniciales (efecto de la antigüedad, las altas o las bajas, etc.) obliga a considerarlos de forma individual para garantizar que las condiciones relativas a los saldos se cumplen para todos ellos. De todos modos, a efectos de modelización, se agrupa a los trabajadores en función de su saldo inicial (aquellos trabajadores que tengan el mismo valor inicial, o parecido) reduciendo así el número de variables y restricciones necesarias y permitiendo afrontar problemas con un número muy elevado de trabajadores.

\section{Datos}

T Número de períodos del horizonte de planificación $(t=1, \ldots, T)$.

E Conjunto ordenado de números de los periodos de producción (excluyendo del horizonte de planificación los periodos en los que los trabajadores están de vacaciones).

P Conjunto de productos.

I $\quad$ Stock de producto $p$ al inicio del horizonte de planificación $(\forall p \in P)$.

$d_{p t} \quad$ Demanda prevista de los producto de tipo $p$ en el periodo $t(\forall p \in P ; t=1, \ldots, T)$.

$\rho_{p} \quad$ Productividad (en unidades/hora) para el producto $p(\forall p \in P)$.

W Conjunto de grupos de trabajadores.

Swo Saldo de la cuenta de horas del grupo de trabajadores $w(\forall w \in W)$ al inicio del horizonte de planificación, que puede ser negativo, positivo o nulo (los trabajadores están agrupados según su saldo inicial).

$N_{w} \quad$ Número de trabajadores que forman parte del grupo $w(\forall w \in W)$.

$\mathrm{S}^{-}, \mathrm{S}^{+} \quad$ Cotas inferior y superior, respectivamente, del saldo de la cuenta de horas; cumpliéndose necesariamente que $S^{-} \leq 0$.

h Número de horas normal o de referencia, por periodo. $h^{-}, h^{+} \quad$ Cotas inferior y superior, respectivamente, del número de horas de trabajo ordinarias por periodo.

$h^{++} \quad$ Cota superior del número de horas de trabajo, por periodo.

u $\quad$ Cota superior, para la duración del horizonte de planificación, del número de horas extra para cada uno de los trabajadores del grupo $w(\forall w \in W)$.

$\hat{u}_{w} \quad$ Cota superior, para la duración del horizonte de planificación, del número de horas extra más el número de horas de overaccount para cada uno de los trabajadores del grupo w $(\forall w \in W)$.

wta $a^{-}$, ta $^{+}$Cotas inferior y superior, respectivamente, del saldo global de la cuenta de horas (i.e., la suma de los saldos de las cuentas de todos los trabajadores) al final del horizonte de planificación.

$C p$ Coste variable unitario de producción para el producto $p(\forall p \in P)$, excluyendo los costes de personal.

$c i$ Coste de tener en stock, durante un periodo, una unidad del producto $p(\forall p \in P)$.

$\mathrm{Cr}_{\mathrm{p}} \quad$ Coste de una unidad de demanda perdida de producto $p(\forall p \in P)$.

$\alpha \quad$ Coste de una hora extra (por trabajador).

$\beta \quad$ Coste de una hora de overaccount (por trabajador). Lógicamente suponemos que $\beta<\alpha$.

$\lambda_{1} \quad$ Penalización por las horas de underaccount (horas perdonadas).

$\lambda_{2} \quad$ Peso del saldo positivo de las cuentas de horas al final del horizonte de planificación.

$\lambda_{3} \quad$ Peso del saldo negativo de las cuentas de horas al final del horizonte de planificación.

\section{Variables}

$q_{p t} \geq 0 \quad$ Unidades producidas de producto $p$ en el periodo $t(\forall p \in P ; \forall t \in E)$. 
$I_{p t} \geq 0 \quad$ Stock de producto $p$ al final del periodo $t$ $(\forall p \in P ; t=1, \ldots, T)$.

$r_{p t} \geq 0 \quad$ Demanda perdida del producto $p$ en el periodo $t(\forall p \in P ; t=1, \ldots, T)$.

$x_{t}^{-}, x_{t}^{+} \geq 0$ Horas de trabajo, por debajo y por encima, respectivamente, del valor de referencia, $h$, en el periodo $t(\forall t \in E)$. Las variables corresponden a las horas regulares, es decir, las que no tienen la consideración de horas extra.

$e_{t} \geq 0 \quad$ Horas extra en el periodo $t(\forall t \in E)$.

$\mathrm{e}_{w t}^{+} \geq 0 \quad$ Horas de overaccount del grupo de trabajadores $w$ en el periodo $t(\forall w \in W, \forall t \in E)$.

$e_{w t}^{-} \geq 0 \quad$ Horas de underaccount del grupo de trabajadores $w$ en el periodo $t(\forall w \in W, \forall t \in E)$.

$c l_{t} \in\{0, I\}$ Indica si en la semana $t$ la compañía cierra debido a baja demanda o no $(\forall t \in E)$.

Swt Saldo de la cuenta de horas del grupo de trabajadores $w$ en el periodo $t(\forall w \in W$, $\forall t \in E$ ); $s_{w t}$ puede tomar valor negativo (que indica que los trabajadores del grupo $w$ deben horas a la compañía), positivo (que indica que la compañía debe horas a los trabajadores) o nulo.

$f^{+}, f^{-} \geq 0$ Balance global (suma de las cuentas de horas de todos los trabajadores) positivo y negativo, respectivamente, al final del horizonte de planificación.

\section{Modelo}

$$
\begin{aligned}
{[M I N] z=} & \sum_{t \in E} \sum_{p \in P} c p_{p} a_{p t}+\sum_{t=1}^{T} \sum_{p \in P}\left(c i_{p} l_{p t}+c r_{p} r_{p t}\right)+ \\
& \sum_{t \in E} \sum_{w \in W} N_{w}\left(\alpha_{e_{t}}+\left(\beta-\frac{t}{|00 \cdot| E \mid}\right) e_{w t}^{+}+\lambda_{\mid} e_{w t}^{-}\right)+ \\
& +\lambda_{2} f^{+}+\lambda_{3} f^{-} \\
q_{p t}+l_{p, t-1}+r_{p t}=d_{p t}+l_{p t} & \forall p \in P ; \forall t \in E \\
I_{p, t-1}+r_{p t}=d_{p t}+I_{p t} & \forall p \in P ; \forall t \notin E \\
\sum_{p \in P} \frac{q_{p t}}{\rho_{p}} \leq h+x_{t}^{+}-x_{t}^{-}+e_{t} & \forall t \in E
\end{aligned}
$$

$$
\begin{array}{ll}
s_{w l}=s_{w 0}+x_{l}^{+}-x_{l}^{-}-e_{w l}^{+}+e_{w l}^{-} & \forall w \in W \\
s_{w t}=s_{w j}+x_{t}^{+}-x_{t}^{-}-e_{w t}^{+}+e_{w t}^{-} & \forall w \in W ; \forall t \in E-\{\mid\} ; \\
& j=\max _{k \in E \mid k<t}(k)
\end{array}
$$$$
\sum_{t \in E} e_{t} \leq u_{w} \quad \forall w \in W
$$$$
\sum_{t \in E}\left(e_{t}+e_{w t}^{+}\right) \leq \hat{u}_{w} \quad \forall w \in W
$$$$
\sum_{w \in W} N_{w} s_{w T}=f^{+}-f^{-}
$$$$
0 \leq f^{+} \leq w t a^{+}
$$$$
0 \leq f^{-} \leq w t a^{-}
$$

$h_{c l_{t}} \leq x_{t}^{-} \leq h-h^{-}\left(1-c_{t}\right) \quad \forall t \in E$

$$
\begin{array}{ll}
0 \leq x_{t}^{+} \leq h^{+}-h & \forall t \in E \\
0 \leq e_{t} \leq h^{++}-h^{+} & \forall t \in E \\
s^{-} \leq s_{w t} \leq s^{+} & \forall w \in W ; \forall t \in E \\
0 \leq e_{w t}^{+} \leq x_{t}^{+} & \forall w \in W ; \forall t \in E ; \forall t \in E \\
0 \leq e_{w t}^{-} \leq x_{t}^{-} & \\
c_{t} \in\{0,1\} & \\
\left.q_{p t t^{\prime}}\right|_{p t,}, r_{p t} \geq 0 &
\end{array}
$$

[I] es la función objetivo que incluye los costes de producción, stock, demanda perdida, horas extra y tiempo del overaccount y del underaccount; [2] y [3] son los saldos de producción, stock, demanda perdida y demanda; [4] expresa que el número de horas de trabajo requerido para la producción no debe ser mayor que las horas disponibles; [5] y [6] expresan, para cada grupo de trabajadores y para cada período, el saldo de la cuenta de horas; [7] y [8] imponen, para cada grupo de trabajadores, las cotas superiores, para la duración del horizonte de planificación, del número de horas extras y el número de horas extras más el número de las horas de overaccount; [9] calcula el saldo global de las cuentas de horas, como la suma de los saldos de las cuentas para todos los trabajadores, al final del horizonte de planificación; [10] y [I I] imponen las cotas superior e inferior, respectivamente, de los saldos globales positivo y negativo de la cuenta de horas, al final del horizonte de planificación; 
[12] impone que si la variable $c_{t}$ toma valor I, entonces las horas trabajadas valen 0 (ya que el número de horas por debajo del valor de referencia h, es igual a h), con lo que la compañía cerraría durante ese período. Si, por el contrario, l $_{t}$ toma valor 0 , entonces se impone la cota superior para el número de horas de trabajo por debajo del valor de referencia. Las ecuaciones [13] a [17] imponen las cotas superior e inferior a las horas de trabajo por encima del valor de referencia, a las horas extra, al saldo de las cuentas de horas, a las horas de overaccount y a las de underaccount, respectivamente. Por último, [1 8] impone el carácter binario de las variables $c_{t}$ y [19] el carácter no negativo de las variables restantes.

En la función objetivo [ I], se ha incluido una modificación en el coste de las horas del overaccount, considerándolas ligeramente más costosas cuanto más cerca están del comienzo del horizonte de planificación. Ello se hace para evitar programar horas de overaccount antes de que sea absolutamente necesario. Sin esta modificación dos horas de overaccount serían equivalentes independientemente de donde estén situadas en el horizonte de planificación.

\section{I. Caso adicional}

Se considera un caso adicional, el de las industrias que trabajan 24 h/día y 5 días/semana y que no pueden alargar la jornada si no es trabajando en sábado y, en este caso, un número mínimo de horas (los trabajadores sólo se desplazaran si deben hacer como mínimo $h_{s}$ horas). Se deben definir nuevos datos y variables:

\section{Datos}

$h_{s} \quad$ Cota inferior de las horas de trabajo por encima del valor de referencia $h$.

Variables

$y_{t} \in\{0,1\}$ Indica si en la semana $t$ el número de horas de trabajo es superior al valor de referencia $h$, o no $(\forall t \in E)$.

Para este caso la restricción expresada en la ecuación [13] se sustituye por las ecuaciones [20] y [2 I], que se definen a continuación:

$$
\begin{array}{ll}
x^{+}{ }_{t} \geq h_{s} y_{t} & \forall t \in E \\
x^{+}{ }_{t} \leq\left(h^{+}-h\right) y_{t} & \forall t \in E
\end{array}
$$

\section{Experiencia computacional}

Se ha realizado una experiencia computacional para evaluar la eficacia del modelo. Globalmente los resultados se pueden considerar satisfactorios.

\section{Referencias}

AZMAT, C.; HURLIMANN, T., and WIDMER, M. (2004). «Mixed integer programming to schedule a single-shift workforce under annualized hours.» Annals of Operations Research 128 (I -4): I 99-2 I 5. COROMINAS, A.; LUSA A., and PASTOR, R. (2002). «Using MILP to plan annualised working hours.» Journal of the Operational Research Society 53 (10): | 101 - I 108.

COROMINAS, A.; LUSA, A., and PASTOR, R. (2007a). «Planning annualised hours with a finite set of weekly working hours and cross-trained workers.» European Journal of Operational Research 176 (I): 230-239.

COROMINAS, A.; LUSA, A., and PASTOR, R. (2007b). «Using a MILP model to establish a framework for an annualised hours agreement.» European Journal of Operational Research 17 7(3): I 495- 1506.

COROMINAS, A.; OJEDA, J., and PASTOR, R. (2005). «Multi-objective allocation of multi-function workers with lower bounded capacity.» Journal of the Operational Research Society 56 (6): 738-743.

COROMINAS, A.; PASTOR, R., and RODRIGUEZ, E. (2006). «Rotational allocation of tasks to multifunctional workers in a service industry.» International Journal of Production Economics 103 ( I): 3-9.

DGT-DIRECCIÓN GENERAL DETRABAJO (2004). Convenio colectivo interprovincial de Renault España, S.A., 2004/2006. Boletín Oficial del Estado de 6 de Julio de 2004. Páginas 24988-25042.

DGT-DIRECCIÓN GENERAL DETRABAJO (2006). Convenio colectivo de XVII Convenio colectivo de la empresa SEAT, SA. Boletín Oficial del Estado de 13 de Marzo de 2006. Páginas I 2059- 12095.

ERNST, A. T.; JIANG, H.; KRISHNAMOORTHY, M., and SIER, D. (2004). «Staff scheduling and rostering:A review of applications, methods and models.» European Journal of Operational Research 153 ( I): 3-27.

HUNG, R. (1999). «Scheduling a workforce under annualized hours.» International Journal of Production Research 37 ( I): 2419-2427.

LEHNDORFF, S. (1999). New working time systems, work organisation and re-distribution of work. Düsseldorf, Institut Arbeit und Technik Abteilung Arbeitsmarkt: 89.

OWEN, J. ( 1 977). «Flexitime: Some Problems and Solutions.» Industrial and Labor Relations Review 30 (2): I52- 160. 
PASTOR, R., and OLIVELLA, J. (2008) «Selecting and adapting weekly work schedules with working time accounts: A case of a retail clothing chain.» European Journal of Operational Research I84 (I): $1-12$.

S. DEL A. (2007). Sony convierte en fijos a 450 trabajadores en su planta de televisiones de Vilade- cavalls. El País. Edición Catalunya de 10 de Enero de 2007.

SPE-SERVICIO PROVINCIAL DE ECONOMÍA, HACIENDA Y EMPLEO (2004). Convenio colectivo de la empresa Opel España de Automóviles, S.L. Boletín Oficial de la provincia de Zaragoza de 9 Julio de 2004. Páginas 4355-4374. 